\title{
A Simple Optical Analysis of Gravitational Lensing
}

\author{
Xing-Hao Ye* and Qiang Lin'ं \\ Department of Physics, Zhejiang University, Hangzhou 310027, China
}

(Dated: November 20, 2018)

\begin{abstract}
We analyzed the influence of static gravitational field on the vacuum and proposed the concept of inhomogeneous vacuum. According to the corresponding Fermat's principle in the general relativity, we derived a graded refractive index of vacuum in a static gravitational field. We found that the light deflection in a gravitational field can be calculated correctly with the use of this refractive index and therefore the gravitational lensing can be treated conveniently with the optical method. For illustration, we simulated the imaging of gravitational lensing, figured out the time delay between the two images and calculated the lens mass in a conventional optical way.
\end{abstract}

PACS numbers: 42.25.Bs, 42.50.Lc, 04 .

\section{INTRODUCTION}

Gravitational lensing is an effect predicted by general relativity [1, 2, 3]. It has been now a powerful tool for the study of the so intractable problems in astrophysics and cosmology such as the value of Hubble constant [4], the physics of quasars [5], the mass and mass distribution of a galaxy or a galaxy cluster[2], the large scale structure of the universe [6, 7], the existence of dark matter [8, 9, 10], the nature of dark energy [11, 12] and so on. Since a curved spacetime is involved in relativistic treatment of the gravitational lensing, inconvenience and intricacy are inevitable. So it is valuable to seek for an alternative method to handle problems of gravitational lensing in a simpler way.

In the framework of curved spacetime, the velocity of light in vacuum is regarded as a constant $c$, which means that vacuum is "homogeneous" and "isotropic", i.e., vacuum does not differ from place to place, and its refractive index is always 1 . However, the recent theoretical and experimental progresses demonstrate that such concept of vacuum turns out to be inappropriate when there are matters or fields within finite distance. For example, the vacuum inside a microcavity is modified due to the existence of the cavity mirrors, which will alter the zero-point energy inside the cavity and cause an attractive force between the two mirrors known as Casimir effect [13, 14], which has been verified experimentally [15, 16]. A second example is that, under the influence of electromagnetic field, vacuum can be polarized, which has led to astonishingly precise agreement between predicted and observed values of the electron magnetic moment and Lamb shift, and may influence the motion of photons [17]. Dupays et al. [18] studied the propagation of light in the neighborhood of magnetized neutron stars. They pointed out that the light emitted by background astronomical objects will be deviated due to the optical properties of quantum vacuum in the presence of a magnetic field. Also in [19], Rikken and Rizzo considered the anisotropy of the optical properties of the vacuum when a static magnetic field $\mathbf{B}_{0}$ and a static electric field $\mathbf{E}_{0}$ are simultaneously applied perpendicular to the direction of

\footnotetext{
*Electronic address yxhow @ 163.com
}

†Electronic address qlin@zju.edu.cn light propagation. They predicted that magnetoelectric birefringence will occur in vacuum under such conditions. They also demonstrated that the propagation of light in vacuum becomes anisotropic with the anisotropy in the refractive index being proportional to $\mathbf{B}_{0} \times \mathbf{E}_{0}$.

The facts that the propagation of light in vacuum can be modified by applying electromagnetic fields to the vacuum implies that the vacuum is actually a special kind of optical medium [17, 18]. This is similar to the Kerr electro-optic effect and the Faraday magneto-optic effect in nonlinear dielectric medium. This similarity between the vacuum and the dielectric medium implies that vacuum must also have its inner structure, which could be influenced by matter or fields as well. Actually, the structure of quantum vacuum has already been investigated in quite a number of papers [20, 21, 22].

So, if we introduce an inhomogeneous vacuum within the framework of flat spacetime instead of a curved spacetime with a homogeneous vacuum, we will then find an equivalent but simpler method, i.e., an optical method, to treat the problem of gravitational lensing. It is just what we will do in the paper below.

\section{THE VACUUM REFRACTIVE INDEX IN A GRAVITATIONAL FIELD}

In order to seek for an optical way to the treatment of gravitational lensing, we will start from a formula representing the corresponding Fermat's principle for the propagation of light in a static gravitational field derived from the general relativity by Landau and Lifshitz [23]:

$$
\delta \int g_{00}{ }^{-1 / 2} d l=0,
$$

where $d l$ is the local length element passed by light and measured by the observer at position $r$ in the gravitational field, $r$ is the distance from this element of light to the center of gravitational matter $M, g_{00}$ is a component of the metric tensor $g_{\mu \nu}, g_{00}^{-1 / 2} d l$ corresponds to an element of optical path length. $g_{00}{ }^{-1 / 2}=d t / d \tau$, where $d \tau$ represents the time interval measured by the local observer for a light ray passing through the length $d l$, while $d t$ is the corresponding time measured by the 


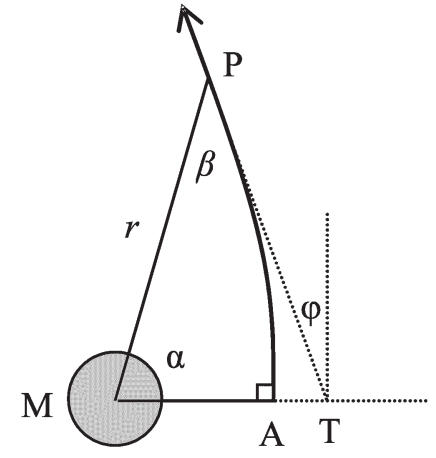

FIG. 1: Light deflection caused by a graded refractive index.

observer at infinity. Eq.(1) could then be rewritten as

$$
\begin{aligned}
\delta \int g_{00}^{-1 / 2} d l & =\delta \int \frac{d t}{d \tau} d l \\
& =\delta \int \frac{d t}{d \tau} \frac{d l}{d s} d s \\
& =\delta \int n d s=0,
\end{aligned}
$$

where $d s$ is the length element measured by the observer at infinity, corresponding to the local length $d l$.

Eq.(2) shows that if we set the scale of length and time at infinity as a standard scale for the whole gravitational space and time, the propagation of light then satisfies the standard representation of Fermat's principle, with the space - actually the vacuum influenced by the gravitational matter - possessing a graded refractive index given by

$$
n=\frac{d t}{d \tau} \frac{d l}{d s}=n_{1} n_{2},
$$

where $n_{1}$ relates to the time transformation relation (i.e., the time dilation effect) $d t / d \tau$ and $n_{2}$ relates to the space transformation relation (i.e., the length contraction effect) $d l / d s$.

Now consider the light deflection caused by this graded refractive index. In Fig.1, curve AP represents the light ray, $\beta$ is the angle between the position vector $\mathbf{r}$ and the tangent at the point $\mathrm{P}$ on the ray, $\alpha$ is the angular displacement of the vector $\mathbf{r}, \varphi$ is the deflection angle of light . For a gravitational matter with spherical symmetry, the refractive index of the vacuum around will also be spherically symmetrical, i.e., depends only on the distance $r$ for a given mass $M$. The light propagation then satisfies the following relation [24]:

$$
n r \sin \beta=\text { constant, }
$$

or

$$
n r \sin \beta=n_{0} r_{0},
$$

where $r_{0}$ and $n_{0}$ represent the radius and refractive index at the nearest point A respectively.

Since

$$
\tan \beta=\frac{r d \alpha}{d r}
$$

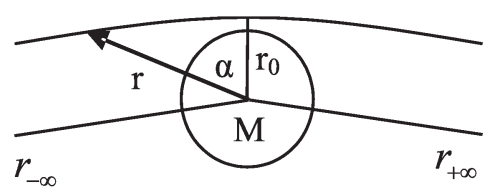

FIG. 2: Light deflection in solar gravitational field.

associating with Eq.(5) reaches

$$
d \alpha=\frac{d r}{r \sqrt{\left(\frac{n r}{n_{0} r_{0}}\right)^{2}-1}} .
$$

While the solution given by the general relativity [1, 25] reads

$$
d \alpha=\frac{d R}{R \sqrt{\left[\left(\frac{R}{R_{0}}\right)^{2}\left(1-\frac{2 G M}{R_{0} c^{2}}\right)+\frac{2 G M}{R c^{2}}\right]-1}},
$$

where $R$, differing from the radius $r$ in the framework of flat spacetime, is the radial coordinate in the Schwarzschild metric.

For an ordinary gravitational system, the gravitational field is not extremely strong, i.e., $G M / r c^{2}<<1$, then we have the following relations satisfying Eqs.(7) and (8):

$$
\begin{aligned}
& R=r e^{\frac{G M}{r c^{2}}} \\
& n=e^{\frac{2 G M}{r c^{2}}} .
\end{aligned}
$$

To verify the above expression of graded vacuum refractive index, we consider a light ray passing by the Sun as shown in Fig.2. The total angular displacement of the radius vector $\mathbf{r}$ is

$$
\Delta \alpha=2 \int_{r_{0}}^{\infty} \frac{d r}{r \sqrt{\left(\frac{n r}{n_{0} r_{0}}\right)^{2}-1}},
$$

where $r_{0}$ represents the nearest distance to the center of the Sun. Substituting Eq.(10) into Eq.(11) gives a solution of first order approximation

$$
\Delta \alpha=\pi+\frac{4 G M}{r_{0} c^{2}} .
$$

Then the total deflection angle of light caused by the solar gravitational field is

$$
\Delta \varphi=\Delta \alpha-\pi=\frac{4 G M}{r_{0} c^{2}},
$$

which is fully consistent with that given by the general relativity [3, 27] and the actual measurements [28], showing that the expression of the vacuum refractive index we gave in Eq.(10) is workable. 


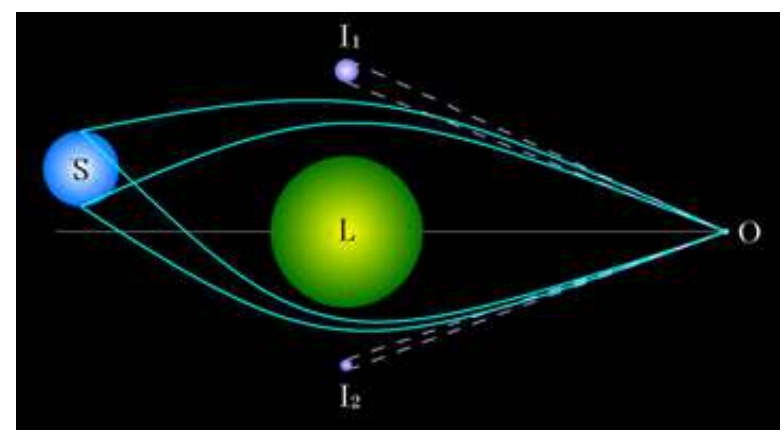

FIG. 3: Light path simulation of a gravitational lensing.

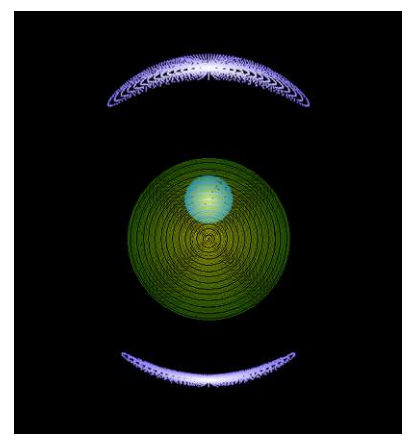

FIG. 4: Image shape simulation of a gravitational lensing.

\section{OPTICAL TREATMENT OF GRAVITATIONAL LENSING}

The deflection of light caused by the gravitational field of celestial bodies leads to the effect of gravitational lensing. Formerly, this effect should be calculated complicatedly with the general relativity [1, 2, 3]. Once we have introduced the concept of graded vacuum refractive index and obtained its relation with mass $M$ and position $r$, the problem of gravitational lensing could then be treated easily with the conventional optical method [29].

Considering a source $S$ and a lens $L$ of mass $M$, the light emitted from $S$ is bent due to the gravitational field of the lens. The bent light could be figured out through Eqs.(4) and (10). Drawing the extension line of the light from the observer $O$, the apparent (observed) position of the image could then be found out. A computer simulated imaging is shown in Fig.3, where the upper image $I_{1}$ and lower image $I_{2}$ are just drawn above and below the lens $L$ for comparison between the three.

In this way, the image shape could be figured out easily. Fig.4 shows a simulated result under the same conditions as that of Fig.3. For comparison, the source (the small circle) is also drawn proportionally in Fig.4, though it is blocked by the intervening lens (the big circle). From the figure, we see that the images are elongated tangentially. If source $S$ is located at axis $O L$, the two images will be interconnected forming a ringlike image named "Einstein ring" (Fig.5). These results are consistent with the known facts [1, 2, 3].

Another use of this optical method is to calculate the time delay between the two images of a gravitational lensing.

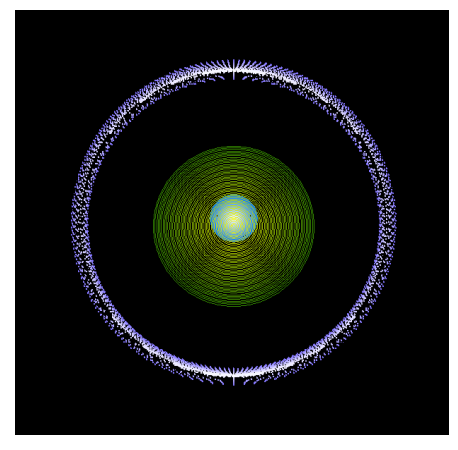

FIG. 5: Einstein ring simulation.

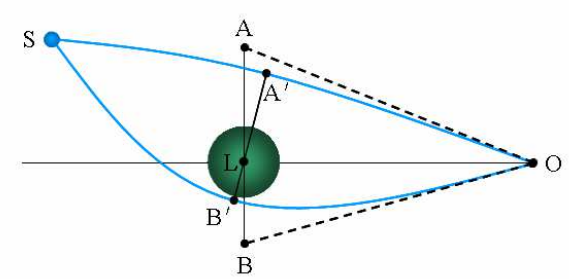

FIG. 6: Time delay between the two images.

According to Fig.1 and Eq.(5), we have

$$
d t=\frac{n d s}{c}=\frac{n d r / \cos \beta}{c}=\frac{n d r}{c \sqrt{1-\left(\frac{n_{0} r_{0}}{n r}\right)^{2}}} .
$$

Then, the time delay between the two images $A$ and $B$ in Fig.6 is

$$
\Delta t=t_{B}-t_{A}=\int_{S B^{\prime} O} d t-\int_{S A^{\prime} O} d t
$$

where $A^{\prime}$ and $B^{\prime}$ are the nearest points to the lens center for the two light paths respectively.

Ordinarily, a gravitational lensing system satisfies

$$
r_{S}\left(\text { and: } r_{O}\right)>>r_{A^{\prime}}\left(\text { and: } r_{B^{\prime}}\right)>>G M / c^{2},
$$

where $r_{S}, r_{O}, r_{A^{\prime}}, r_{B^{\prime}}$ represent the distances from points $S, O$, $A^{\prime}, B^{\prime}$ to the lens center respectively.

Using Eqs.(10), (14), (15) and (16) we get

$$
\Delta t=\frac{4 G M}{c^{3}} \ln \frac{r_{A^{\prime}}}{r_{B^{\prime}}} .
$$

For example, in the case of the first observed gravitational lensing — the binary quasar Q0957+561, the measured time delay is $\Delta t=417 \pm 3$ days [4, 30].

This time delay could be used to estimate the mass of the lens galaxy:

$$
M=\frac{c^{3} \Delta t}{4 G \ln \frac{r_{A^{\prime}}}{r_{B^{\prime}}}} \approx \frac{c^{3} \Delta t}{4 G \ln \frac{\beta_{A O L}}{\beta_{B O L}}} .
$$

In the case of $\mathrm{Q} 0957+561, \beta_{A O L} / \beta_{B O L} \approx 5.5$, thus we get $M \approx 2 \times 10^{42} \mathrm{~kg}$. Since the mass of our Milky Way galaxy is 
about $3.6 \times 10^{41} \mathrm{~kg}$, the mass we figured out here is an appropriate estimation. Considering that the lens galaxy has a mass distribution and image B is located among this distribution, the actual value of the lens mass will be a little larger.

The result we obtained above can be further used to estimate the Hubble constant. A simplified calculation for the cases of Q0957+561, HE2149-2745 and RXJ1131-1231 [30] gives an average value $H_{0} \approx 70 \mathrm{~km} / \mathrm{s} \cdot \mathrm{Mpc}$.

\section{CONCLUSIONS}

We have proposed the concept of inhomogeneous vacuum with graded refractive index based on the analysis of the influence of static gravitational field on the vacuum. we derived the expression of this refractive index through the general relativity and the optical principle, and verified it in the effect of light deflection caused by a gravitational field. By using this expression, we investigated the gravitational lensing in a conventional optical way and showed some computer simulations for the imaging of gravitational lensing. Also in this way, we figured out the time delay between the two images of a gravitational lensing and gave a simple calculation of the lens mass, which could be further used to find out the value of Hubble constant. The results indicate that, the concept of inhomogeneous vacuum is mathematically equivalent to the curved spacetime in the general relativity. In addition to the equivalence and convenience, the investigation of an inhomogeneous vacuum (actually a quantum vacuum) instead of a curved spacetime also promises an approach to the physical connection between the general relativity and the quantum mechanics.

\section{Acknowledgments}

We wish to acknowledge the supports from the National Key Project for Fundamental Research (grant no. 2006CB921403), the National Hi-tech project (grant no. 2006 AA06A204) and the Zhejiang Provincial Qian-Jiang-Ren-Cai Project of China (grant no. 2006R10025).
[1] S. Mollerach and E. Roulet, Gravitational Lensing and Microlensing (World Scientific, New Jersey, 2002).

[2] J. Wambsganss, Gravitational Lensing in Astronomy, www.livingreviews.org/Articles/Volume1/1998-12wamb.

[3] H. C. Ohanian and R. Ruffini, Gravitation and Spacetime (W. W. Norton and Company Inc., New York, 1994).

[4] T. Kundić et al., ApJ. 482, 75 (1997).

[5] P. Solomon et al., Nature 426, 636 (2003).

[6] R. Massey et al., Nature 445, 286 (2007).

[7] V. Springel, C. S. Frenk, and S. D. M. White, Nature 440, 1137 (2006).

[8] K.C. Freeman, Science 302, 1902 (2003).

[9] N. Inada et al., Nature 426, 810 (2003).

[10] D. M. Wittman et al., Nature 405, 143 (2000).

[11] C. L. Bennett, Nature 440, 1126 (2006).

[12] C. L. Bennett, Science 307, 879 (2005).

[13] H. Gies and K. Klingmüller, Phys. Rev. Lett. 96, 220401 (2006).

[14] T. Emig et al., Phys. Rev. Lett. 96, 080403 (2006).

[15] S. K. Lamoreaux, Phys. Rev. Lett. 78, 5 (1997).

[16] H. B. Chan et al., Science 291, 1941 (2001).

[17] N. Ahmadi and M. Nouri-Zonoz, Phys. Rev. D. 74, 044034 (2006).
[18] A. Dupays et al., Phys. Rev. Lett. 94, 161101 (2005).

[19] G. L. J. A. Rikken and C. Rizzo, Phys. Rev. A. 63, 012107 (2000) ; 67, 015801 (2003).

[20] A. Armoni, A. Gorsky, and M. Shifman, Phys. Rev. D. 72, 105001 (2005).

[21] A. Barroso et al., Phys. Rev. D. 74, 085016 (2006) .

[22] K. R. Dienes, E. Dudas, and T. Gherghetta, Phys. Rev. D. 72, 026005 (2005).

[23] L. D. Landau and E. M. Lifshitz, The Classical Theory of Fields (Pergamon Press, New York, 1975).

[24] M. Born and E. Wolf, Principles of Optics (7th edition) (Cambridge University Press, Cambridge, 1999).

[25] P. Amore and S. Arceo, Phys. Rev. D. 73, 083004 (2006).

[26] H. A. Lorentz, A. Einstein, and H. Minkowski, The Principle of Relativity (Methuen, London, 1923).

[27] S. Weinberg, Gravitation and Cosmology (John Wiley and Sons, New York, 1972).

[28] E. B. Fomaleont and R. A. Sramek, Phys. Rev. Lett. 36, 1475 (1976).

[29] X. H. Ye and Q. Lin, http://arxiv.org/abs/0704.1173v1

[30] http://www.cfa.harvard.edu/glensdata/ 